\title{
Predicting associations between microRNAs and target genes in breast cancer by bioinformatics analyses
}

\author{
TIANYING ZHENG, XING ZHANG, YONGGANG WANG and XIUCUI YU
}

\begin{abstract}
Department of Chemotherapy, Cancer Center, Qilu Hospital of Shandong University, Jinan, Shandong 250012, P.R. China
\end{abstract}
Received April 22, 2015; Accepted May 26, 2016

DOI: $10.3892 / \mathrm{ol} .2016 .4731$

\begin{abstract}
Breast cancer is the leading type of cancer among females. However, the association between microRNAs (miRNAs) and target genes in breast tumorigenesis is poorly studied. The original data set GSE26659 was downloaded from the Gene Expression Omnibus, and then the differentially expressed miRNAs among 77 breast cancer patients and 17 controls were identified using the Limma package in $\mathrm{R}$ software. Furthermore, breast cancer-related differentially expressed miRNAs were selected from a human miRNA disease database and their target genes were selected from five miRNA databases. Then, functional analysis was performed for the target genes followed by construction of a miRNA-target gene network. A total of 34 differentially expressed miRNAs were identified, including 13 breast cancer-related miRNAs. Moreover, the target genes of the 13 miRNAs were significantly enriched in regulation of transcription $(\mathrm{P}=7.43 \mathrm{E}-09)$ and pathways related to cancer $(\mathrm{P}=3.33 \mathrm{E}-11)$. Finally, eight upregulated miRNAs (including hsa-miR-425) and five downregulated miRNAs (including hsa-miR-143, hsa-miR-145 and hsa-miR-125b) were identified in the miRNA-target gene network. In conclusion, using bioinformatics approaches, we demonstrate that the changes in regulation of transcription and cancer pathways may play significant roles in the process of breast cancerogenesis. Differentially expressed miRNAs and their target genes may be new targets for breast cancer therapy.
\end{abstract}

\section{Introduction}

Breast cancer is the most common malignancy in females worldwide, and the disease is often fatal due to metastasis dissemination (1). The disease develops from breast tissue with symptoms including a lump in the breast, dimpling of the skin, a red scaly patch of skin, changes in breast shape or

Correspondence to: Dr Xiucui Yu, Department of Chemotherapy, Cancer Center, Qilu Hospital of Shandong University, 107 West Wenhua Road, Jinan, Shandong 250012, P.R. China

E-mail: xiucuiyuuyu@163.com

Key words: breast cancer, differentially expressed microRNA, functional analysis, protein-protein interaction network fluid coming from the nipple (2). Generally, the first noticeable symptom of breast cancer is a lump that feels different from the rest of the breast tissue. Several factors, including smoking tobacco, lack of physical exercise, ionizing radiation, obesity, and having children late in life or not at all, may induce breast cancer (3). The survival rates of breast cancer in developing countries are poor, with 1.68 million cases and 522,000 mortalities in 2012 (4). Therefore, numerous researchers and physicians are focusing on the pathogenesis of the disease with the aim of improving current therapies and identifying new treatments.

microRNAs (miRNAs) are small non-coding RNA molecules, which participate in post-transcriptional gene regulation in a sequence-specific manner. It has been suggested that they are involved in the establishment and progression of human tumors, appearing to be critical biomarkers of cancer (5). For instance, miR-148b is a major coordinator of breast cancer progression in a relapse-associated miRNA signature by targeting integrin $\alpha 5$, Rho-associated coiled-coil containing protein kinase 1 (ROCK1), and phosphatidylinositol-4,5-bisphosphate 3-kinase, catalytic subunit $\alpha$ (PIK3CA) (5). miR-335 was observed to inhibit proliferation, cell cycle progression, colony formation and invasion by targeting paired box 6 (PAX6) in breast cancer cells (6). In addition, miR-185 suppresses tumor proliferation by directly targeting E2F transcription factor 6 (E2F6) and indirectly upregulating breast cancer 1 , early onset (BRCAl) in triple-negative breast cancer (7). Although the pathogenesis of breast cancer has been investigated, the mechanism is not fully understood. More crucial miRNAs that might play significant roles in breast cancerogenesis need to be identified.

Therefore, in our present study, the underlying mechanisms of breast cancer were further explored and predicted by bioinformatics approaches, including screening breast cancer-related miRNAs, predicting target genes of the miRNAs, performing functional analysis for target genes, and constructing a protein-protein interaction network. The findings may provide new insights into breast cancer pathogenesis and bring about novel targets for cancer therapy.

\section{Materials and methods}

Microarray data. The Gene Expression Omnibus database (GEO, http://www.ncbi.nlm.nih.gov/geo/) in the National Center for Biotechnology Information (NCBI) is currently 
the largest fully public gene expression resource, and includes 214,268 samples and 4,500 platforms (8). In the present study, microarray data set GSE26659 (5) was downloaded from GEO, which included 77 gene chips from ductal breast carcinoma biopsies and 17 from mammoplasties. The 77 frozen tumor specimens were selected from the Tumor Bank of the Department of Obstetrics and Gynecology at the University of Turin, Italy. They were obtained from patients who were diagnosed with invasive breast cancer at $\mathrm{T}$ and $\mathrm{N}$ stages and underwent primary surgical treatment between 1988 and 2001 at a median age of 54 years. The 17 frozen mammoplasty samples were from the École polytechnique fédérale (Swiss Federal Institute of Technology), Lausanne, Switzerland, and were included in the study as normal breast controls. Raw data were collected based on the platform GPL8227 Agilent-019118 Human miRNA Microarray 2.0 G4470B (miRNA ID version; Affymetrix Inc., Santa Clara, CA, USA).

Data preprocessing and differential analysis. The original data and annotation files were downloaded and normalized. Then according to the SOFT formatted family files, the normalized data series matrix files were mapped to their corresponding miRNA names. The expression values of multiple probes for a given miRNA were reduced to a single value by taking the average expression value. The Affy package (9) in R (https://www.r-project.org/) was selected for background correction, normalization and calculation of expression values. After that, the linear models for microarray analysis (Limma) package (10) in R was used to calculate the probability of miRNAs being differentially expressed between cases and controls. The fold change (FC) and its logarithm value ( $\log \mathrm{FC}$ ) were also determined. A false discovery rate $(\mathrm{FDR})<0.01$ and $\log \mathrm{FCl}>1$ were set as the cut-offs to screen differentially expressed miRNAs.

Identification of breast cancer-related differentially expressed miRNAs. The selected differentially expressed miRNAs were mapped into the human miRNA disease database (HMDD; http://cmbi.bjmu.edu.cn/hmdd and http://202.38.126.151/ $\mathrm{hmdd} /$ tools/hmdd2.html) to further select the differentially expressed miRNAs related to breast cancer. As a database for experimentally supported human miRNA and disease associations, HMDD serves as a valuable resource for investigating the roles of miRNAs in human disease (11).

Target gene prediction. The target genes of breast cancer-related differentially expressed miRNAs were predicted by five miRNA databases, namely miRanda (http://microrna.sanger.ac.uk) (12), MirTarget2 (http://nar. oxfordjournals.org/cgi/content/abstract/34/5/1646) (13), PicTar (http://pictar.bio.nyu.edu) (14), PITA (http://genie.weizmann.ac.il/pubs/mir07) (15) and TargetScan (http://targetscan. org) (16). In order to obtain more solid results, target genes that occurred in not less than three databases were regarded as the target genes of breast cancer-related differentially expressed miRNAs. In addition, the published oncogenes and suppressors of breast cancer were selected from TSGene (http://bioinfo.mc.vanderbilt.edu/TSGene/) (17) and Tumor Associated Gene (TAG; http://www.binfo.ncku.edu.tw/TAG/) databases (18).
Functional enrichment analysis. The Database for Annotation, Visualization and Integrated Discovery (DAVID) bioinformatics resource consists of an integrated biological knowledge base and analytic tools aimed at systematically extracting biological meaning from large gene or protein lists (19). In the present study, DAVID was applied to conduct Kyoto encyclopedia of genes and genomes (KEGG) pathway and gene ontology (GO) enrichment analyses for the identified target genes. KEGG is a knowledge base for systematic analysis of gene functions (20). GO analysis predicts the function of the target genes in three aspects, including biological processes, cellular components and molecular function (21). $\mathrm{P}<0.05$ and $\mathrm{FDR}<0.05$ were set as thresholds.

miRNA-target gene network construction. Based on the identified breast cancer-related differentially expressed miRNAs and their target genes, the miRNA-target gene network was constructed and visualized using Cytoscape (http://www. cytoscape.org/) (22), which is open source software used for visualizing biological network and integrating data.

\section{Results}

Differentially expressed miRNAs. Among the 77 breast cancer samples and 17 controls, a total of 34 differentially expressed miRNAs were screened out, which included 19 upregulated and 15 downregulated miRNAs in breast cancer patients (Table I). By mapping them to HMDD, 13 of these were selected as breast cancer-related differentially expressed miRNAs; namely hsa-let-7d, hsa-let-7e, hsa-let-7g, hsa-miR-100, hsa-miR-125b, hsa-miR-143, hsa-miR-145, hsa-miR-155, hsa-miR-197, hsa-miR-21, hsa-miR-223, hsa-miR-425 and hsa-miR-497.

Target genes of breast cancer-related miRNAs. The target genes of the 13 miRNAs were identified from the Miranda, MirTarget2, PicTar, PITA and TargetScan databases. Finally, 3,086 target genes of these 13 miRNAs were screened from not less than three databases. Among these genes, 47 oncogenes [including zinc finger protein 217 (ZNF217), Wolf-Hirschhorn syndrome candidate 1 (WHSC1) and ubiquitin specific peptidase 6(USP6)] and 123 tumor suppressor genes (including ZIC family member 1 (ZIC1), zinc finger homeobox 3 ( $Z F H X 3)$ and wingless-type MMTV integration site family, member $5 \mathrm{~A}$ (WNT5A)] were further filtered by combining the TSGene and TAG databases (Table II).

Functional analysis for target genes. The top five pathways and GO terms are listed in Tables III and IV, respectively. In Table III, the most significant KEGG pathway was hsa05200, pathways in cancer $(\mathrm{P}=3.33 \mathrm{E}-11)$ and 76 target genes, including $E 2 F 2$, fibroblast growth factor 9 ( $F G F 9$ ) and $W N T 3 A$, were enriched in this pathway. Moreover, 60, 36, 26 and 23 differentially expressed miRNAs affected the MAPK signaling pathway $(\mathrm{P}=2.02 \mathrm{E}-08)$, neurotrophin signaling pathway $(\mathrm{P}=3.42 \mathrm{E}-08)$, colorectal cancer $(\mathrm{P}=1.10 \mathrm{E}-06)$ and chronic myeloid leukemia $(\mathrm{P}=6.37 \mathrm{E}-06)$, respectively (Table III). Additionally, the most significant GO term was GO:0045449, regulation of transcription ( $\mathrm{P}=7.43 \mathrm{E}-13$; Table IV). A total of 360 target genes, including 
Table I. List of differentially expressed genes with $\log \mathrm{FCl}>1$ and FDR $<0.01$.

\begin{tabular}{|c|c|c|c|c|}
\hline Category & miRNA ID & $\log \mathrm{FC}$ & P-value & FDR \\
\hline \multirow[t]{19}{*}{ Upregulated } & hsa-miR-21 & 3.068525 & $5.10 \mathrm{E}-31$ & $6.12 \mathrm{E}-29$ \\
\hline & hsa-miR-142-3p & 2.128498 & $5.73 \mathrm{E}-10$ & $2.46 \mathrm{E}-09$ \\
\hline & hsa-miR-155 & 2.087345 & $1.35 \mathrm{E}-14$ & $2.32 \mathrm{E}-13$ \\
\hline & hsa-miR-15b & 1.991570 & $4.72 \mathrm{E}-22$ & $2.83 \mathrm{E}-20$ \\
\hline & hsa-miR-425 & 1.950364 & $3.00 \mathrm{E}-13$ & $2.77 \mathrm{E}-12$ \\
\hline & hsa-miR-342-3p & 1.807487 & $1.77 \mathrm{E}-09$ & $6.84 \mathrm{E}-09$ \\
\hline & hsa-miR-331-3p & 1.672587 & $8.82 \mathrm{E}-20$ & $2.65 \mathrm{E}-18$ \\
\hline & hsa-miR-125a-5p & 1.616122 & $4.28 \mathrm{E}-12$ & $2.85 \mathrm{E}-11$ \\
\hline & hsa-miR-130b & 1.581679 & $5.43 \mathrm{E}-12$ & $3.43 \mathrm{E}-11$ \\
\hline & hsa-miR-98 & 1.521842 & $1.72 \mathrm{E}-12$ & $1.37 \mathrm{E}-11$ \\
\hline & hsa-let-7e & 1.510875 & $3.49 \mathrm{E}-14$ & $5.20 \mathrm{E}-13$ \\
\hline & hsa-miR-197 & 1.447683 & $1.51 \mathrm{E}-11$ & 8.64E-11 \\
\hline & hsa-miR-223 & 1.385350 & $3.56 \mathrm{E}-08$ & $1.19 \mathrm{E}-07$ \\
\hline & hsa-miR-455-3p & 1.256636 & $2.83 \mathrm{E}-06$ & 7.37E-06 \\
\hline & hsa-let-7d & 1.210423 & $1.21 \mathrm{E}-14$ & $2.32 \mathrm{E}-13$ \\
\hline & hsa-let-7g & 1.161949 & $2.53 \mathrm{E}-13$ & $2.53 \mathrm{E}-12$ \\
\hline & hsa-miR-150 & 1.106677 & 0.000447 & 0.000851 \\
\hline & hsa-let-7f & 1.061395 & $1.83 \mathrm{E}-12$ & $1.37 \mathrm{E}-11$ \\
\hline & hsa-miR-16 & 1.040802 & $2.48 \mathrm{E}-13$ & $2.53 \mathrm{E}-12$ \\
\hline \multirow[t]{15}{*}{ Downregulated } & hsa-miR-130a & -1.025820 & $4.14 \mathrm{E}-08$ & $1.34 \mathrm{E}-07$ \\
\hline & hsa-miR-939 & -1.078370 & $8.47 \mathrm{E}-10$ & 3.39E-09 \\
\hline & hsa-miR-143 & -1.089560 & $3.02 \mathrm{E}-06$ & 7.70E-06 \\
\hline & kshv-miR-K12-3 & -1.127510 & 2.79E-09 & $9.85 \mathrm{E}-09$ \\
\hline & hsa-miR-768-3p & -1.277500 & $6.53 \mathrm{E}-13$ & $5.60 \mathrm{E}-12$ \\
\hline & hsa-miR-101 & -1.295530 & $9.80 \mathrm{E}-14$ & $1.18 \mathrm{E}-12$ \\
\hline & hsa-miR-188-5p & -1.342530 & $2.76 \mathrm{E}-09$ & $9.85 \mathrm{E}-09$ \\
\hline & hsa-miR-497 & -1.385440 & $3.74 \mathrm{E}-12$ & $2.64 \mathrm{E}-11$ \\
\hline & hsa-miR-26a & -1.408450 & $3.90 \mathrm{E}-14$ & $5.20 \mathrm{E}-13$ \\
\hline & hsa-miR-125b & -1.419680 & $5.30 \mathrm{E}-10$ & 2.35E-09 \\
\hline & hsa-miR-100 & -1.432230 & $4.55 \mathrm{E}-10$ & $2.10 \mathrm{E}-09$ \\
\hline & hsa-miR-99a & -1.560150 & $6.26 \mathrm{E}-09$ & $2.15 \mathrm{E}-08$ \\
\hline & hsa-miR-140-3p & -1.822440 & $2.00 \mathrm{E}-21$ & $7.99 \mathrm{E}-20$ \\
\hline & hsa-miR-923 & -2.289380 & $2.18 \mathrm{E}-10$ & $1.09 \mathrm{E}-09$ \\
\hline & hsa-miR-145 & -2.552090 & $2.89 \mathrm{E}-15$ & $6.94 \mathrm{E}-14$ \\
\hline
\end{tabular}

FC, fold change; FDR, false discovery rate.

myocyte enhancer factor $2 \mathrm{C}(M E F 2 C), M E F 2 A$ and growth differentiation factor $6(G D F 6)$ were enriched in this GO term. Enzyme-linked receptor protein signaling pathway $(\mathrm{P}=7.45 \mathrm{E}-11)$, nucleoplasm $(\mathrm{P}=1.18 \mathrm{E}-10)$, positive regulation of transcription $(\mathrm{P}=1.96 \mathrm{E}-10)$ and nuclear lumen $(\mathrm{P}=2.22 \mathrm{E}-10)$ were the next four most significant GO terms after regulation of transcription (Table IV).

Construction of miRNA-target gene network. With the correlations between the breast cancer-related differentially expressed miRNAs and their target genes, a miRNA-target gene network was constructed using Cytoscape. In Fig. 1, interactions between eight upregulated miRNAs (including hsa-miR-425) and five downregulated miRNAs (including
hsa-miR-143, hsa-miR-145 and hsa-miR-125b) and their various target genes are shown.

\section{Discussion}

Like other cancers, breast cancer occurs due to interactions between an environmental factor and a genetically susceptible host. In the present study, the underlying mechanism of the disease was analyzed by using a series of bioinformatics approaches. Gene expression profile including 77 breast cancer samples and 17 controls was used in our study, and 34 differentially expressed miRNAs were identified. Furthermore, breast cancer-related miRNAs that may play roles in breast cancer development and progression were selected from HMDD. 
Table II. Oncogenes and tumor suppressor genes among target genes of breast cancer-related differentially expressed microRNAs.

\begin{tabular}{ll}
\hline Category & \multicolumn{1}{c}{ Genes } \\
\hline Oncogenes & BCL11A, BCL2L2, CBL, CCNA2, CCND1, CCND2, CCNE1, CHKA, CRK, CRKL, CSF1R, \\
& DEK, EIF5A2, ELK1, ERBB3, ERG, FGF2, FGFR1, FGFR3, FOS, GNAS, HMGA2, \\
& HOXA10, KRAS, LMO2, MAP3K8, MCF2, MYBL1, MYCL1, NET1, NRAS, PDGFRA, \\
& PIM1, PTPN11, RAF1, RALA, RUNX1T1, SALL4, SERTAD2, SET, SKI, TAF15, TAL1, \\
& TRIM32, USP6, WHSC1, ZNF217 \\
Tumor suppressor genes & ADAMTS18, AKAP12, APAF1, APC, ARHGAP20, ARHGAP35, ARHGEF12, ARID1A, \\
& ARID2, ARID3B, AXIN2, BACH2, BHLHE41, BLCAP, BTG2, CADM1, CADM4, \\
& CAMTA1, CBFA2T3, CDC73, CDKN1B, CDKN2A, CHEK1, CREBL2, CSMD1, CTDSPL, \\
& CTNND1, CUL2, CUL5, CYGB, CYLD, DAB2, DAPK1, DDX3X, DIRAS1, DKK3, \\
& EGLN1, EGR1, ENC1, EPB41L3, ERF, FOXO1, FOXO3, FOXP1, HBP1, IGF2R, IGFBP3, \\
& IGFBP5, ITGB3, JUP, KIF1B, KLF5, KRIT1, LATS2, LIN9, LOX, LRIG3, LRP1B, MFHAS1, \\
& MTAP, MTSS1, MTUS1, NF2, NR4A3, NRCAM, OPCML, PAFAH1B1, PDCD4, PDS5B, \\
& PHF6, PHLDA3, PHLPP2, PPAP2A, PPP1CA, PPP2R1B, PRDM1, PRDM4, PRKAR1A, \\
& PTCH1, PTPN2, PTPN3, PTPRD, RARB, RASL10B, RASSF5, RBM6, RCN2, RECK, \\
REV3L, RINT1, RND3, RSRC2, SASH1, SERPINB5, SIAH1, SLC39A1, SMAD2, SMAD3, & SMARCA4, SOCS1, SOX11, SPRY2, ST5, STARD13, TCF4, TGFBI, TGFBR2, TGFBR3, \\
TIMP3, TOPORS, TP53, TP53INP1, TSC1, TUSC2, UBE4B, UHRF2, UNC5A, VCL, \\
WHSC1L1, WIF1, WNT5A, ZFHX3, ZIC1
\end{tabular}

Table III. Top five significant pathways regulated by target genes of breast cancer-related differentially expressed microRNAs .

\begin{tabular}{lllr}
\hline Category & \multicolumn{1}{c}{ Term } & Count & P-value \\
\hline KEGG_PATHWAY & hsa05200:Pathways in cancer & 76 & $3.33 \mathrm{E}-11$ \\
KEGG_PATHWAY & hsa04010:MAPK signaling pathway & 60 & $2.02 \mathrm{E}-08$ \\
KEGG_PATHWAY & hsa04722:Neurotrophin signaling pathway & 36 & $3.42 \mathrm{E}-08$ \\
KEGG_PATHWAY & hsa05210:Colorectal cancer & 26 & $1.10 \mathrm{E}-06$ \\
KEGG_PATHWAY & hsa05220:Chronic myeloid leukemia & 23 & $6.37 \mathrm{E}-06$ \\
\end{tabular}

FDR, false discovery rate. Count indicates target genes enriched in the pathway $(\mathrm{P}<0.05$ and FDR $<0.05)$.

A total of 13 breast cancer-related differentially expressed miRNAs, including let-7d, miR-100 and miR-125b, were identified. Moreover, the target genes of these 13 miRNAs were predicted from five databases, and the miRNA-target gene network containing eight upregulated miRNAs (including miR-21, miR-197, let-7g, let-7e and let-7d) and five downregulated miRNAs (including miR-143, miR-145 and miR-100) was visualized. The let-7 family is a tumor-suppressor gene family, which is often inactivated in human cancers, including breast cancer. As one of the target genes of the let-7 family, lin-28 homolog A (LIN28) has been reported to promote tumorigenic activity by suppressing let-7 miRNA maturation in breast cancer cells (23). miR-100 has been reported to inhibit tumorigenesis in oral squamous cell carcinoma, ovarian cancer and breast cancer by interfering with proliferation and survival signaling (24-26). Additionally, miR-143 and miR-145 have been observed to synergistically regulate $\mathrm{v}$-erb-b2 avian erythroblastic leukemia viral oncogene homolog 3 to suppress cell proliferation and invasion in breast cancer (27). Moreover, miR-197 and miR-21 were noted to be prominently upregulated in male breast cancer (28). The deregulation of these miRNAs and target genes is associated with breast tumorigenesis, and our results are consistent with these studies, indicating that these miRNAs and their target genes might be potential therapeutic targets for breast cancer treatment.

Furthermore, following functional analysis, target genes were identified to be notably enriched in regulation of transcription $(\mathrm{P}=7.43 \mathrm{E}-13)$ and pathways related to cancer $(\mathrm{P}=3.33 \mathrm{E}-11)$. The biological process associated with regulation of transcription means any process that modulates the frequency, rate or extent of cellular DNA-templated transcription. A total of 380 target genes, including $M E F 2 C, M E F 2 A$ and $G D F 6$, were enriched in this GO term. $M E F 2 C$ is involved in cardiac morphogenesis, myogenesis and vascular development. $M E F 2 C$, which is regulated by let- $7 \mathrm{~g}$, has been 
Table IV. Top five significant gene ontology terms enriched by target genes of breast cancer-related differentially expressed microRNAs.

\begin{tabular}{|c|c|c|c|c|}
\hline Category & Term & Count & P-value & FDR \\
\hline GOTERM_BP_FAT & GO:0045449 regulation of transcription & 380 & 7.43E-13 & $1.37 \mathrm{E}-09$ \\
\hline GOTERM_BP_FAT & $\begin{array}{l}\text { GO:0007167 enzyme-linked receptor } \\
\text { protein signaling pathway }\end{array}$ & 78 & $7.45 \mathrm{E}-11$ & $1.37 \mathrm{E}-07$ \\
\hline GOTERM_CC_FAT & GO:0005654 nucleoplasm & 143 & $1.18 \mathrm{E}-10$ & $1.74 \mathrm{E}-07$ \\
\hline GOTERM_BP_FAT & $\begin{array}{l}\text { GO:0045941 positive regulation of } \\
\text { transcription }\end{array}$ & 110 & $1.96 \mathrm{E}-10$ & $3.62 \mathrm{E}-07$ \\
\hline GOTERM_CC_FAT & GO:0031981 nuclear lumen & 209 & $2.22 \mathrm{E}-10$ & $3.26 \mathrm{E}-07$ \\
\hline
\end{tabular}

FDR, false discovery rate. Count indicates target genes enriched in the pathway. BP and CC are biological processes and cellular component $(\mathrm{P}<0.05$ and FDR $<0.05)$.

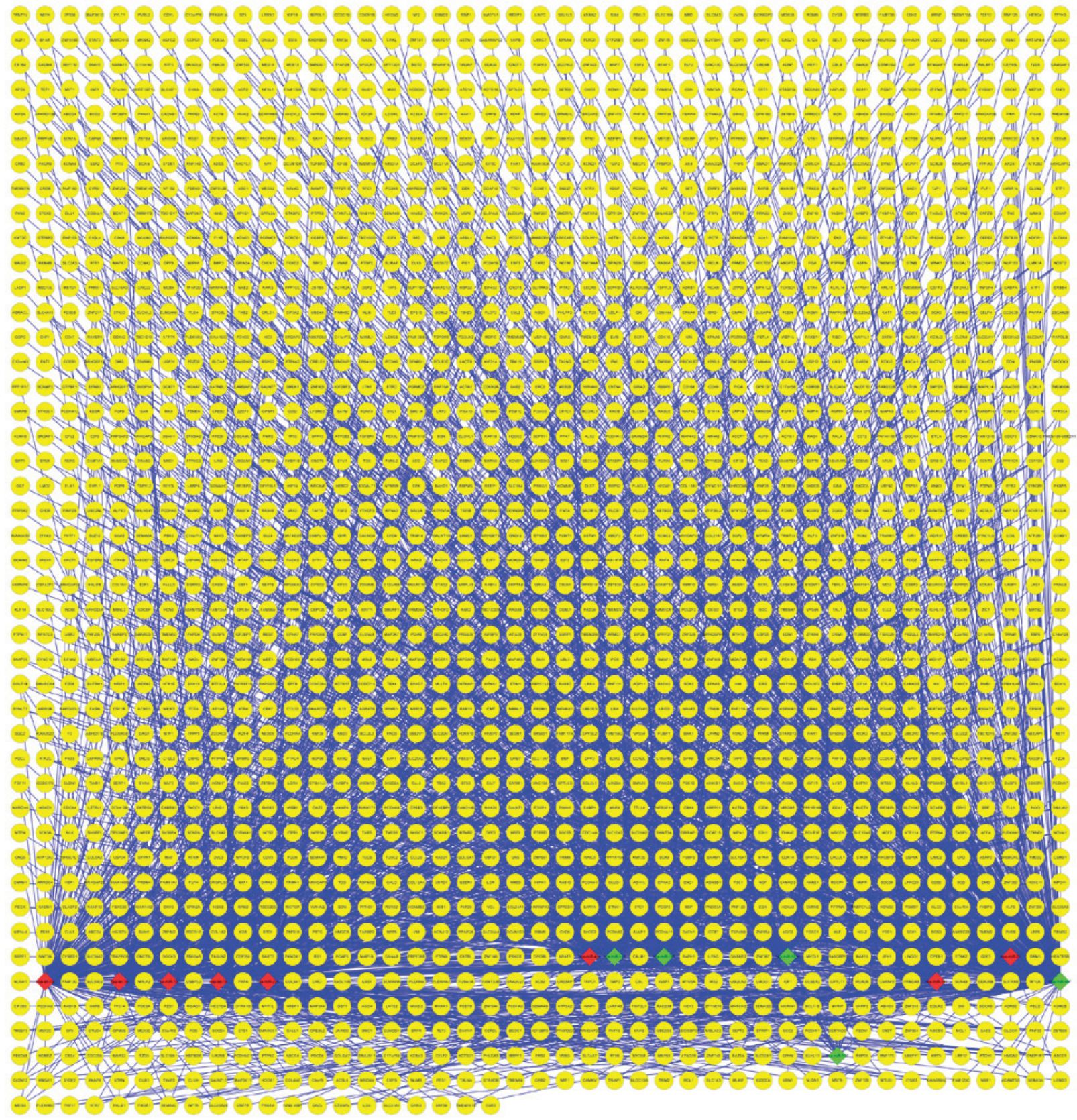

Figure 1. The protein-protein interaction network between breast cancer-related differentially expressed microRNAs (miRNAs) and their target genes. The red and green diamonds represent up- and downregulated miRNAs, respectively. Yellow circles are target genes of miRNAs. 
reported as the main regulator in primary breast cancer (29). Mutations and deletions of this gene have been associated with severe mental retardation, stereotypic movement, epilepsy and cerebral malformation (30). MEF $2 A$, regulated by miR-155, is involved in vertebrate skeletal muscle development and differentiation (31). Another target gene, GDF6, is associated with growth and differentiation of developing embryos, and is involved in early regulation of cell growth and development (32). In the miRNA-target gene network, GDF6 was regulated by several miRNAs, including let-7d. Taken together, these results might be useful in developing breast cancer treatments.

Additionally, 76 target genes, including E2F2, FGF9 and WNT3A, regulated pathways related to cancer. As a target of the transcription proteins of small DNA tumor viruses, transcription factor E2F2 controls the cell cycle and the action of tumor suppressor proteins. In particular, miR-155 has been reported to regulate tumor development and metastasis in a mouse model of metastatic breast cancer by targeting E2F2 (33). FGF9, regulated by let-7e, is associated with epithelial-to-mesenchymal transition and invasion by inducing vascular endothelial growth factor expression (34). WNT3A has been implicated in oncogenesis, adipogenesis, regulation of cell fate and patterning during embryogenesis. However, the roles of FGF9 and WNT3A in breast cancer have not been investigated. In breast tumorigenesis, the up- or downregulated expression of these miRNAs and genes may affect cancer pathways, and then induce the disease.

Our results provide new insights into the pathogenesis and treatment of breast cancer. The disturbed cancer-related pathways and regulation of transcription may play significant roles in breast cancer development. Moreover, $M E F 2 A$, GDF6, FGF9 and WNT3A may be new targets for breast cancer therapy. These findings may be useful to researchers and physicians in future studies. However, due to the limitations of bioinformatics approaches, the results in our study are predicted, and the findings are not confirmed by traditional bio-molecular methods. Therefore, further genetic and experimental studies with a larger sample size are necessary to verify these results.

\section{References}

1. Liu S, Clouthier SG and Wicha MS: Role of microRNAs in the regulation of breast cancer stem cells. J Mammary Gland Biol Neoplasia 17: 15-21, 2012.

2. Wang F, Li H, Tan PH, Chua ET, Yeo RM, Lim FL, Kim SW, Tan DY and Wong FY: Validation of a nomogram in the prediction of local recurrence risks after conserving surgery for Asian women with ductal carcinoma in situ of the breast. Clin Oncol (R Coll Radiol) 26: 684-691, 2014.

3. Fortune-Greeley AK, Wheeler SB, Meyer AM, Reeder-Hayes KE, Biddle AK, Muss HB and Carpenter WR: Preoperative breast MRI and surgical outcomes in elderly women with invasive ductal and lobular carcinoma: a population-based study. Breast Cancer Res Treat 143: 203-212, 2014.

4. Hansen BT, Nygård M, Falk RS and Hofvind S: Breast cancer and ductal carcinoma in situ among women with prior squamous or glandular precancer in the cervix: a register-based study. Br J Cancer 107: 1451-1453, 2012.

5. Cimino D, De Pittà C, Orso F, Zampini M, Casara S, Penna E, Quaglino E, Forni M, Damasco C, Pinatel E, et al: miR148b is a major coordinator of breast cancer progression in a relapse-associated microRNA signature by targeting ITGA5, ROCK1, PIK3CA, NRAS and CSF1. FASEB J 27: 1223-1235, 2013.
6. Meng Y,Zou Q, Liu T, Cai X, Huang Y and Pan J: microRNA-335 inhibits proliferation, cell-cycle progression, colony formation, and invasion via targeting PAX6 in breast cancer cells. Mol Med Rep 11: 379-385, 2015

7. Tang H, Liu P, Yang L, Xie X, Ye F, Wu M, Liu X, Chen B, Zhang L and Xie X: miR-185 suppresses tumor proliferation by directly targeting E2F6 and DNMT1 and indirectly up-regulating BRCA1 in triple negative breast cancer. Mol Cancer Ther 13: 3185-3197, 2014.

8. Barrett T, Wilhite SE, Ledoux P, Evangelista C, Kim IF, Tomashevsky M, Marshall KA, Phillippy KH, Sherman PM, Holko M, et al: NCBI GEO: archive for functional genomics data sets-update. Nucleic Acids Res 41 (Database Issue): D991-D995, 2013.

9. Wilson CL and Miller CJ: Simpleaffy: a BioConductor package for Affymetrix Quality Control and data analysis. Bioinformatics 21: 3683-3685, 2005.

10. Gautier L, Cope L, Bolstad BM and Irizarry RA: affy - analysis of Affymetrix GeneChip data at the probe level. Bioinformatics 20: 307-315, 2004.

11. Li Y, Qiu C, Tu J, Geng B, Yang J, Jiang T and Cui Q: HMDD v2.0: a database for experimentally supported human microRNA and disease associations. Nucleic Acids Res 42 (Database Issue): D1070-D1074, 2014.

12. Enright AJ, John B, Gaul U, Tuschl T, Sander C and Marks DS: MicroRNA targets in Drosophila. Genome Biol 5: R1, 2003.

13. Wang $X$ and El Naqa IM: Prediction of both conserved and nonconserved microRNA targets in animals. Bioinformatics 24: 325-332, 2008 .

14. Krek A, Grün D, Poy MN, Wolf R, Rosenberg L, Epstein EJ, MacMenamin P, da Piedade I, Gunsalus KC, Stoffel M and Rajewsky N: Combinatorial microRNA target predictions. Nature Genet 37: 495-500, 2005.

15. Kertesz M, Iovino N, Unnerstall U, Gaul U and Segal E: The role of site accessibility in microRNA target recognition. Nature Genet 39: 1278-1284, 2007.

16. Lewis BP, Shih IH, Jones-Rhoades MW, Bartel DP and Burge CB: Prediction of mammalian microRNA targets. Cell 115: 787-798, 2003.

17. Zhao M, Sun J and Zhao Z: TSGene: A web resource for tumor suppressor genes. Nucleic Acids Res 41 (Database Issue): D970-D976, 2013.

18. Chen JS, Hung WS, Chan HH, Tsai SJ and Sun HS: In silico identification of oncogenic potential of fyn-related kinase in hepatocellular carcinoma. Bioinformatics 29: 420-427, 2013.

19. Huang DW, Sherman BT, Tan Q, Collins JR, Alvord G, Roayaei J, Stephens R, Baseler MW, Lane HC and Lempicki RA: The DAVID gene functional classification tool: a novel biological module-centric algorithm to functionally analyze large gene lists. Genome Biol 8: R183, 2007.

20. Kanehisa M and Goto S: KEGG: Kyoto encyclopedia of genes and genomes. Nucleic Acids Res 28: 27-30, 2000.

21. Tran NT and Huang CH: Gene expression and gene ontology enrichment analysis for $\mathrm{H} 3 \mathrm{~K} 4 \mathrm{me} 3$ and $\mathrm{H} 3 \mathrm{~K} 4 \mathrm{me} 1$ in mouse liver and mouse embryonic stem cell using ChIP-Seq and RNA-Seq. Gene Regul Syst Bio 8: 33-43, 2014.

22. Smoot ME, Ono K, Ruscheinski J, Wang PL and Ideker T: Cytoscape 2.8: New features for data integration and network visualization. Bioinformatics 27: 431-432, 2011.

23. Sakurai M, Miki Y, Masuda M, Hata S, Shibahara Y, Hirakawa H, Suzuki T and Sasano H: LIN28: a regulator of tumor-suppressing activity of let-7 microRNA in human breast cancer. J Steroid Biochem Mol Biol 131: 101-106, 2012.

24. Gebeshuber CA and Martinez J: miR-100 suppresses IGF2 and inhibits breast tumorigenesis by interfering with proliferation and survival signaling. Oncogene 32: 3306-3310, 2013.

25. Henson BJ, Bhattacharjee S, O'Dee DM, Feingold E and Gollin SM: Decreased expression of miR-125b and miR-100 in oral cancer cells contributes to malignancy. Genes Chromosomes Cancer 48: 569-582, 2009.

26. Nagaraja AK, Creighton CJ, Yu Z, Zhu H, Gunaratne PH, Reid JG, Olokpa E, Itamochi H, Ueno NT, Hawkins SM, et al: A link between mir-100 and FRAP1/mTOR in clear cell ovarian cancer. Mol Endocrinol 24: 447-463, 2010.

27. Yan X, Chen X, Liang H, Deng T, Chen W, Zhang S, Liu M, Gao X, Liu Y,Zhao C, et al: miR-143 and miR-145 synergistically regulate ERBB3 to suppress cell proliferation and invasion in breast cancer. Mol Cancer 13: 220, 2014. 
28. Lehmann U, Streichert T, Otto B, Albat C, Hasemeier B Christgen H, Schipper E, Hille U, Kreipe HH and Länger F: Identification of differentially expressed microRNAs in human male breast cancer. BMC Cancer 10: 109, 2010.

29. Baca-López K, Mayorga M, Hidalgo-Miranda A, Gutiérrez-Nájera N and Hernández-Lemus E: The role of master regulators in the metabolic/transcriptional coupling in breast carcinomas. PLoS One 7: e42678, 2012.

30. Sturtzel C, Testori J, Schweighofer B, Bilban M and Hofer E: The transcription factor MEF $2 \mathrm{C}$ negatively controls angiogenic sprouting of endothelial cells depending on oxygen. PloS One 9: e101521, 2014.

31. Zhou Y, Liu Y, Jiang X, Du H, Li X and Zhu Q: Polymorphism of chicken myocyte-specific enhancer-binding factor $2 \mathrm{~A}$ gene and its association with chicken carcass traits. Mol Biol Rep 37: $587-594,2010$
32. Banka S, Cain SA, Carim S, Daly SB, Urquhart JE, Erdem G, Harris J, Bottomley M, Donnai D, Kerr B, et al: Leri's pleonosteosis, a congenital rheumatic disease, results from microduplication at 8q22.1 encompassing GDF6 and SDC2 and provides insight into systemic sclerosis pathogenesis. Ann Rheum Dis 74: 1249-1256, 2015.

33. Bollig-Fischer A, Marchetti L, Mitrea C, Wu J, Kruger A, Manca V and Drăghici S: Modeling time-dependent transcription effects of HER2 oncogene and discovery of a role for E2F2 in breast cancer cell-matrix adhesion. Bioinformatics 30: 3036-3043, 2014.

34. Teishima J, Yano S, Shoji K, Hayashi T, Goto K, Kitano H, Oka K, Nagamatsu H and Matsubara A: Accumulation of FGF9 in prostate cancer correlates with epithelial-to-mesenchymal transition and induction of VEGF-A expression. Anticancer Res 34: 695-700, 2014. 